\title{
Surgical Strategy for Spondylodiscitis Due to Candida Albicans in an Immunocompromised Host
}

\author{
-Case Report-
}

\author{
Tsutomu TOKUYAMA, Shigeru NISHIZAWA, Naoki YOKOTA, Seiji OHTA, \\ Tetsuo YOKOYAMA, and Hiroki NAMBA
}

Department of Neurosurgery, Hamamatsu University School of Medicine, Hamamatsu, Shizuoka

\begin{abstract}
A 44-year-old woman receiving systemic chemotherapy for cerebellar medulloblastoma developed thoracolumbar spondylodiscitis due to Candida albicans associated with abscesses in the bilateral psoas muscles. As long-term medical therapy with fluconazole was not effective, radical removal of the affected lesions and anterior bone grafting were performed. Corpectomy of the infected vertebra with autologous bone grafting and removal of the psoas muscle were performed via the right transthoracic retroperitoneal approach. Additional posterior instrumentation was not used. Two years after the operation, the patient was doing well, and systemic chemotherapy for medulloblastoma has restarted. Corpectomy with radical resection of surrounding infectious tissues for $C$. albicans spondylodiscitis in an immunocompromised host should be performed when conservative medical treatment is not successful. Further instrumentation surgery might be necessary to prevent further deformity of the spine as the second surgery.
\end{abstract}

Key words: Candida albicans, immunocompromised host, spinal instrumentation, spondylodiscitis, surgery

\section{Introduction}

Spondylodiscitis due to Candida albicans is very rare, ${ }^{7)}$ despite the increase in cases of systemic candidiasis in recent decades due to the high incidence of immunocompromised hosts caused by extensive chemotherapy for malignant tumors or human immunodeficiency virus infection. ${ }^{1,3,5-8)}$ The clinical and radiological findings are nonspecific, ${ }^{7,8)}$ so unawareness and/or misdiagnosis may delay the required management. Antifungal chemotherapy is the first choice, and such medical treatment is generally effective. ${ }^{7-10)}$

We treated a patient developed thoracolumbar spondylodiscitis due to $C$. albicans during repeated systemic chemotherapy for cerebellar medulloblastoma. Long-term antifungal medical therapy was not effective, so we performed radical surgery of total corpectomy of the infected vertebral bodies with anterior autologous bone grafting.

Received December 19, 2001; Accepted May 7, 2002

\section{Case Report}

A 44-year-old woman underwent total removal of cerebellar medulloblastoma at the age of 35 years. She was then given extended focal irradiation up to $60 \mathrm{~Gy}$ and total spinal irradiation up to $32 \mathrm{~Gy}$, followed by repeated systemic chemotherapy. Three years ago, the patient refused continuation of systemic chemotherapy and selected dietary treatment at a local hospital. The dietary treatment included placing a venous catheter in the right subclavian vein for delivery of intravenous hyperalimentation. Subsequently, the patient developed high fever, and C. albicans was detected from blood culture due to inappropriate management of a central venous catheter. She was treated with oral administration of fluconazole for 8 months. The patient complained of back pain, dysesthesia in the bilateral lower extremities, and unsteady gait, and she was readmitted to our hospital.

Neurological examination disclosed sensory disturbance and weakness in the bilateral lower extremities, and bladder dysfunction, which were considered due to myelopathy. Magnetic resonance 

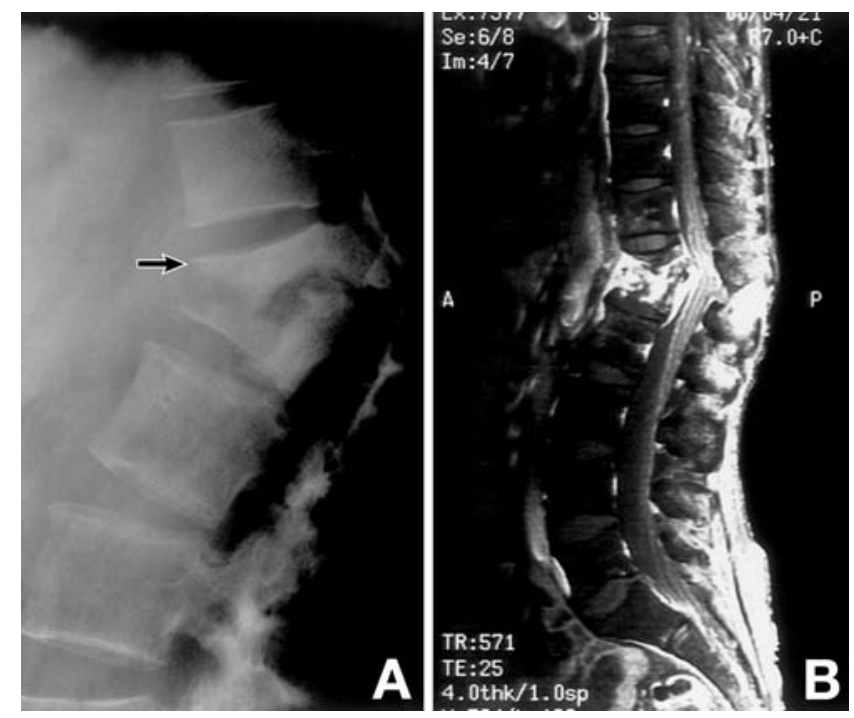

Fig. 1 A: Lateral thoracolumbar radiograph showing marked destructive change of the T12-L1 vertebral bodies (arrow). B: $T_{1}$ weighted magnetic resonance image showing enhancement of the damaged T-12 and L-1 vertebral bodies.

(MR) imaging showed collapse of the L-1 vertebral body, and seeding of the tumor in the intracranial cisterns and spinal subarachnoid space. According to these findings, the patient agreed to further systemic chemotherapy.

Four months after readmission, repeated MR imaging disclosed hypointensity of the L-1 vertebral body, which was enhanced by gadolinium. The patient complained of increasing lumbar pain. Radiography disclosed the lesion had extended to the T-12 vertebral body and T12-L1 intervertebral disc (Fig. 1). Aspiration biopsy was performed under fluoroscopic control, and pus-like discharge was obtained. Microbiological culture identified C. albicans. The diagnosis was spondylodiscitis due to $C$. albicans.

Systemic chemotherapy for medulloblastoma was stopped and high-dose intravenous injection of fluconazole was continued for one month, although the lumbar pain could not be controlled. Computed tomography (CT) disclosed the lesion extended further into the bilateral psoas muscles (Fig. 2). Radical surgery for the infectious lesion was planned, because the fluconazole treatment could not control the infectious lesion, and systemic chemotherapy had to be restarted for the dissemination of medulloblastoma.

The abscess in the right psoas muscle was dominant, so surgery was performed in the right

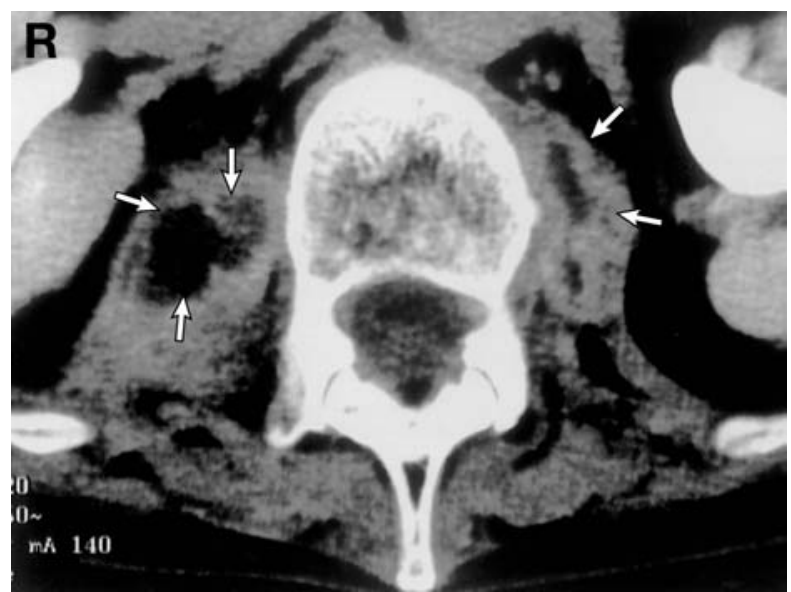

Fig. 2 Computed tomography scan showing abscesses in the bilateral psoas muscles (arrows). The abscess on the right was dominant.

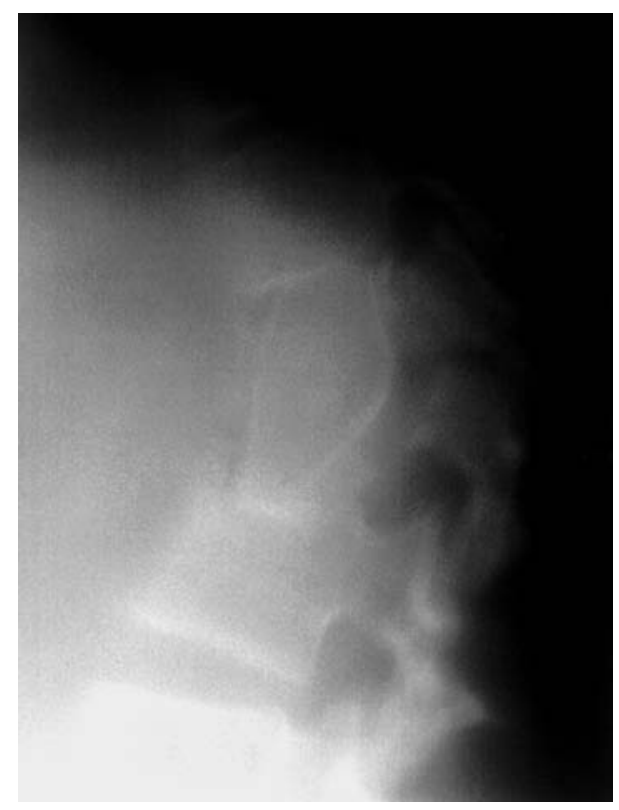

Fig. 3 Postoperative lateral tomography scan showing the transplanted iliac bone graft between T-11 and L-2. No spinal instrumentation was used.

lateral position. The vertebral bodies of T-12 and L-1 were exposed via the right transthoracic retroperitoneal approach, then the right psoas muscle was resected. Total corpectomy of T-12 and L-1 was performed. The iliac bone graft was transplanted without internal fixation by spinal instrumentation (Fig. 3).

The patient was treated with itraconazole pre- and 


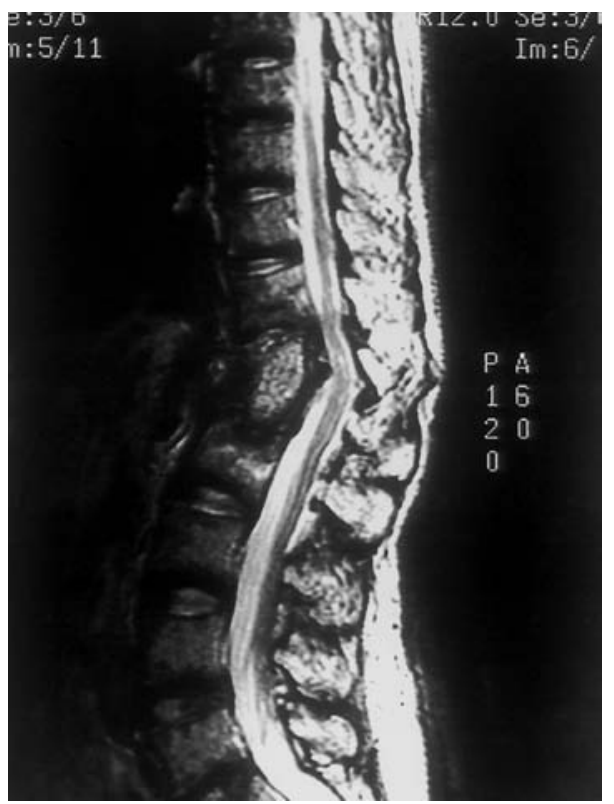

Fig. 4 Postoperative $\mathrm{T}_{2}$-weighted magnetic resonance image showing decompression of the spinal cord, although some kyphotic deformity still remained.

postoperatively and was kept lying flat in a plaster cast bed for 2 months, then she was gradually rehabilitated with a hard brace. After the operation, her back pain, bladder dysfunction, and dysesthesia gradually subsided. The muscle power in the right leg was slightly weakened because of resection of the right psoas muscle. The patient became ambulatory with a cane, and was discharged 3 months after the operation. Postoperative CT disclosed disappearance of the paravertebral abscess. MR imaging showed decompression of the spinal cord, although some spinal kyphotic change still remained (Fig. 4). Oral administration of itraconazole was continued, and systemic chemotherapy was restarted at the outpatient clinic.

\section{Discussion}

Past history of candidemia is a most important clue for the correct diagnosis because of the nonspecific clinical and neuroradiological features. ${ }^{7,8}$ Fungal infection should be considered in an immunocompromised patient who complains of back pain with any changes in the vertebral and/or disc regions on MR imaging. Aspiration biopsy under fluoroscopic control should be performed to confirm the diagnosis.

Antifungal treatment with amphotericin B, fluconazole, itraconazole, 5-fluorocytosine, or ketoconazole, or a combination of these drugs is the first choice for the treatment of spondylodiscitis due to C. albicans. Such medical treatment is generally effective. $^{7-10)}$ However, surgical treatment should be considered in a patient with neurological deficits, extensive collapse of the vertebral bodies, or persistent infection in spite of antifungal chemotherapy. ${ }^{1,11)}$ Furthermore, radical surgery for the infectious lesions is necessary in an immunocompromised host, such as a patient requiring extensive chemotherapy to control malignant tumor.

The direction of approach depends on the dominant side of infection. ${ }^{1)}$ The right transthoracic retroperitoneal approach was used in our case to reach the thoracolumbar vertebral columns because the abscess in the right psoas muscle was dominant. The retroperitoneal approach is very effective to easily reach the thoracolumbar vertebral columns, ${ }^{2,4,13)}$ and also to prevent infection from spreading into any other spaces by surgery.

We extensively removed the right psoas muscle containing the abscess and the T-12 and L-1 vertebral bodies with anterior bone grafting using iliac bone. As we did not use any additional instrumentation, the patient was kept lying flat for 2 months. During the active phase of the infection, we could not assess the extent of infection in the vertebral columns. Use of posterior fixation carries some risk of exacerbation of infection. However, it is possible that posterior instrumentation should be used for spinal stabilization even in cases of infectious lesion, and posterior spinal fixation with instrumentation has a beneficial effect. ${ }^{1,12)}$ In our case, we adopted the classical surgical procedure for fungal infectious spinal lesion. The postoperative result has so far been favorable, so our initial surgical strategy might be justified as the first step of the treatment. However, the spinal deformity still remained after the surgery, and the patient certainly suffered from long-term bed rest following the surgery. Therefore, internal fixation using spinal instrumentation may be required for this patient at an early stage under continuous antifungal treatment.

Recently, percutaneous aspiration and drainage has been effective for acute pyogenic spondylodiscitis. ${ }^{11)}$ This new surgical technique is considered less invasive compared with the conventional open surgical method. However, percutaneous aspiration and drainage failed to improve a patient with spondylodiscitis due to $C$. albicans. ${ }^{6}$ ) Therefore, the usefulness for fungal infection remains unclear.

Fungal spondylodiscitis should be considered as a serious complication in an immunocompromised patient complaining of back pain. Aspiration biopsy and microbiological analysis are required for the 
correct diagnosis. A patient with a malignant tumor which requires systemic chemotherapy should probably undergo early radical surgery instead of prolonged antifungal chemotherapy.

\section{References}

1) Broner FA, Garland DE, Zigler JE: Spinal infections in the immunocompromised host. Orthop Clin North Am 27: 37-46, 1996

2) Burrington JD, Brown C, Wayne ER, Odom J: Anterior approach to the thoraco-lumbar spine: technical considerations. Arch Surg 111: 456-463, 1976

3) Collet P, Biron P, Larbre JP, Llorca G, Lejeune E: [Candida spondylodiscitis. Report of 2 personal cases and 28 cases from the literature]. Rev Med Interne 10: 413-419, 1989 (Fre, with Eng abstract)

4) Cooper PR, Errico TJ, Martin R, Crawford B, DiBartolo T: A systemic approach to spinal reconstruction after anterior decompression for neoplastic disease of the thoracic and lumbar spine. Neurosurgery 32: 1-8, 1993

5) de la Torre Lima J, Jimenez-Mejias ME, de Francisco JL, Martin-Sanz V, Viciana Fernandez JM, Canas Garcia-Otero E: [Candida albicans spondylodiscitis]. Med Clin (Barc) 100: 461-463, 1993 (Spa, with Eng abstract)

6) Derkinderen P, Bruneel F, Bouchaud O, Regnier B: Spondylodiscitis and epidural abscess due to Candida albicans. Eur Spine J 9: 72-74, 2000

7) Hennequin C, Bouree P, Hiesse C, Dupont B, Charpentier B: Spondylodiscitis due to Candida al- bicans: report of two patients who were successfully treated with fluconazole and review of the literature. Clin Infect Dis 23: 176-178, 1996

8) Lafont A, Olive A, Gelman M, Roca-Burniols J, Cots $\mathrm{R}$, Carbonell J: Candida albicans spondylodiscitis and vertebral osteomyelitis in patients with intravenous heroin drug addiction. Report on 3 new cases. J Rheumatol 21: 953-956, 1994

9) Nagata K, Ohashi T, Ariyoshi M, Sonoda K, Imoto H, Inoue A: Percutaneous suction aspiration and drainage for pyogenic spondylitis. Spine 23: 1600-1606, 1998

10) Nguyen TB, Galezowski N, Crouzet J, Laroche F, Blanche P, Herreman G: Spondylodiscitis due to Candida species. J Rheumatol 26: 237-239, 1999

11) Rossel P, Schonheyder HC, Nielsen H: Fluconazole therapy in Candida albicans spondylodiscitis. Scand J Infect Dis 30: 527-530, 1998

12) Wisneski RJ: Infectious disease of the spine: Diagnostic and treatment considerations. Orthop Clin North Am 22: 491-501, 1991

13) Yonenobu K, Korkusuz F, Hosono N, Ebara S, Ono K: Lateral rhachotomy for thoracic spinal lesions. Spine 15: 1121-1125, 1990

Address reprint requests to: S. Nishizawa, M.D., Department of Neurosurgery, Hamamatsu University School of Medicine, 1-20-1 Handayama, Hamamatsu, Shizuoka 431-3192, Japan. e-mail: nisizawa@hama-med.ac.jp. 